\title{
Microsurgical Reconstruction of the Lower Extremity
}

\author{
William C. Pederson, MD, FACS ${ }^{1}$ Luke Grome, MD ${ }^{1}$ \\ ${ }^{1}$ Division of Plastic Surgery, Baylor College of Medicine, Houston, \\ Texas \\ Semin Plast Surg 2019;33:54-58.
}

\begin{abstract}
Address for correspondence William C. Pederson, MD, FACS, Head, Hand and Microsurgery, Texas Children's Hospital, Sam Stal Endowed Professor of Plastic Surgery, Professor of Surgery, Orthopedic Surgery, Neurosurgery, and Pediatrics, Baylor College of Medicine, Houston, TX (e-mail: wcpeders@texaschildrens.org).
\end{abstract}

\begin{abstract}
Keywords

- lower extremity reconstruction

- microsurgery

- functional muscle transfer

- free fibula

- trauma

Reconstruction of bony and soft tissue defects of the lower extremity has been revolutionized by the advent of microsurgical tissue transfer. There are numerous options for reconstruction. Possibilities include transfer of soft tissue, composite (bone and soft tissue) tissue, and functional muscle. Many lower extremity reconstructions require staged procedures. Planning is of paramount importance especially in regard to vascular access when multiple free flaps are required. Soft tissue reconstruction of the lower extremity may be accomplished with muscle flaps such as the rectus femoris and latissimus dorsi covered with a skin graft. Fasciocutaneous flaps such as the anterolateral thigh flap may be more appropriate in a staged reconstruction which requires later elevation of the flap. Loss of a significant portion of bone, such as the tibia, can be difficult to manage. Any gap greater than $6 \mathrm{~cm}$ is considered a reasonable indication for vascularized bone transfer. The contralateral free fibula is the donor site of choice. Functional reconstruction of the anterior compartment of the leg may be performed with a gracilis muscle transfer, effectively eliminating foot drop and providing soft tissue coverage. Muscle tensioning is critical for effective excursion and dorsiflexion of the foot. Long-term results of microsurgical reconstruction of the lower extremity show good results and reasonable rates of limb salvage.
\end{abstract}

Reconstruction of bony and soft tissue defects of the lower extremity has been revolutionized by the advent of microsurgical tissue transfer. We have the ability to repair and reconstruct significant tissue loss due to tumor extirpation or trauma with microsurgical techniques. There are numerous options for soft tissue reconstruction, with the possibility of composite (bone and soft tissue) tissue transfer and functional muscle transfer. This monograph will cover the basics of lower extremity reconstruction with microsurgery.

\section{Planning}

Perhaps the most difficult aspect of performing complex lower extremity reconstruction is proper planning. Singlestage reconstruction of bone and soft tissue defects is often ideal ${ }^{1}$ but may not be practical depending on the situation. If the problem can be taken care of in one stage, planning is less important, but if it is going to take multiple stages (soft tissue, bone, function, etc.), then proper planning is paramount. It is not uncommon to have to perform a soft tissue flap as a first stage to get the wound closed and to prepare the bed for either bone transport or vascularized bone transfer. Vascular access in terms of multiple free flaps (e.g., soft tissue and then bone) is extremely important to avoid "burning bridges" for future flaps. At times, the same recipient vessels can be utilized for more than one free flap, but this may and may not be wise. Frequently, the anterior tibial vessels are divided at the site of injury or fracture, and while these vessels are sometimes not ideal, they can be utilized in an end-to-end fashion for the first flap. The second flap (usually the free fibula) can be anastomosed end to side to the posterior tibial vessels to avoid compromise to the blood flow to the foot. Or this can be done in the opposite fashion, with the original flap anastomosed to the posterior tibial vessels end to side and the fibula anastomosed to the anterior tibial vessels. But keep in mind that the veins accompanying
Issue Theme Free Tissue Transfer Reconstruction; Guest Editor: Yadranko Ducic, MD, FRCS(C), FACS
Copyright $\odot 2019$ by Thieme Medical Publishers, Inc., 333 Seventh Avenue, New York, NY 10001, USA. Tel: +1(212) 584-4662. ISSN 1535-2188. 
the anterior tibial artery are quite small, and may not be appropriate for anastomosis to the large peroneal veins coming off the free fibula. In this case, venous anastomosis to the saphenous or even lesser saphenous veins might be most appropriate.

The choice of the initial soft tissue flap can be important as well. As we discuss later, muscle or fasciocutaneous flaps can be utilized in coverage of lower extremity defects. If there is simply a soft tissue defect, either flap can be entirely appropriate. If there will definitely be a second procedure, whether it be bony or other reconstruction, it is usually easier to secondarily raise a fasciocutaneous flap and/or operate through it than it is to do so through a muscle flap covered with skin graft.

Timing of reconstruction remains an area in which most would agree with Godina that early coverage is the best, ${ }^{2}$ and recent studies have confirmed that coverage within 72 hours lowers rates of flap loss and long-term infection. ${ }^{3}$ Practically speaking, however, this may not be possible in many cases. Negative pressure wound therapy as a temporizing measure until wound coverage can be obtained is probably a reasonable approach in situations where early flap coverage is not possible. $^{4}$

\section{Soft Tissue}

The lower leg tolerates major trauma poorly, as the skin coverage over the tibia is thin, particularly distally. Trauma here frequently leads to an open wound with exposed tibia, usually with an exposed fracture. Conversely, the thigh has a tremendous amount of muscle surrounding the bone, and rarely is soft tissue reconstruction necessary in this area. The upper tibial area can often be covered with local muscle flaps (the soleus or gastrocnemius), but the middle to lower tibial area and the ankle usually will require microvascular tissue transfer for adequate soft tissue reconstruction.

For microvascular tissue transfer to the lower leg, consideration of which recipient vessel to utilize is critical. With significant trauma, the vessels to the lower leg can be injured, leading to issues of vascular access for microsurgical tissue transfer. If the dorsalis pedis and posterior tibial pulse are palpable at the level of the ankle, we feel that vascular studies are probably unnecessary. On the contrary, if both pulses are not palpable, we think it is prudent to obtain vascular studies. Arteriography is usually the best type of study to obtain, computed tomography angiography and/or magnetic resonance angiography may be acceptable depending on the expertise and skill of the local radiologists. In general, however, we feel that the posterior tibial artery is the best choice for anastomosis in most cases. It is protected from trauma due to its position in front of the soleus and injury to this vessel is unusual. The saphenous vein can be accessed with a medial lower leg incision along with the posterior tibial artery, which gives another option for venous drainage of the flap. The anterior tibial artery on the other hand sits on the interosseous membrane and is usually injured with fracture of the tibia and fibula. If the posterior tibial artery is the only vessel supplying the foot, end-to-side anastomosis can be performed without compromising distal circulation.
Anastomosis out of the zone of injury is ideal, but at times difficult to do with extensive trauma. Experience has shown that microvascular anastomosis in the zone of trauma can be successful if the vessels are not badly damaged. ${ }^{5}$ In our experience, the veins pose much more problems than the arteries, and the surgeon should have a backup plan for venous outflow if the chosen veins are damaged and/or thrombosed. Vein grafting to proximal undamaged vessels should always be a consideration in the lower extremity. Another option is to place an arteriovenous vascular loop prior to tissue transfer and utilize this for anastomosis to vessels well out of the zone of injury. ${ }^{6}$

There are a myriad of choices in terms of flaps for coverage of the lower extremity. Traditionally, muscle flaps (i.e., latissimus dorsi, rectus femoris) have been utilized in the lower extremity, but fasciocutaneous flaps (i.e., anterolateral thigh [ALT] flap) have become more popular recently. ${ }^{7}$ Muscle flaps have the advantage that they atrophy over time and contour very nicely ( - Fig. 1). Even when covered with a split-thickness skin graft, the overall appearance is not bad. Muscle flaps may be more difficult to raise again later for further reconstruction (bone grafting, transport, etc.), however. Fasciocutaneous flaps can be too bulky when placed on the lower leg, and will thicken if the patient gains weight. They are much easier to raise and operate through in the future, however. ${ }^{8}$ The best options for the lower extremity in terms of soft tissue coverage are the latissimus dorsi and the ALT flap. Both have long pedicles, can provide adequate tissue for large defects, and the ALT can be utilized with a portion of the fascia lata to reconstruct tendon as well (i.e., the Achilles) (-Figs. 2, 3). The downside of the latissimus is that the patient must be turned to harvest it, and it is generally taken without skin and covered with split-thickness skin graft. The downside of the ALT is that the pedicle dissection can be tedious, and there are a few occasions where an adequate pedicle is not present. Recent work has shown that salvage rates of fasciocutaneous flaps and muscle flaps with a skin paddle are higher than muscle covered with splitthickness skin grafts, probably due to easier monitoring of flow to the flap. ${ }^{9}$

\section{Bone}

Due to the poor skin coverage discussed earlier, tibial fractures are frequently open, which can lead to devascularization of the bone and potentially infection with loss of substance. High-energy fractures and those around the ankle can be difficult to heal even in the best of circumstances. Loss of a significant portion of the tibia can be difficult to manage, and any gap greater than $6 \mathrm{~cm}$ is considered a reasonable indication for vascularized bone transfer, as well as in the face of recalcitrant nonunion and infection (often seen together) (-Fig. 4). This is generally accomplished with microvascular transfer of the contralateral fibula. ${ }^{10,11}$ While the ipsilateral fibula can be moved over to reconstruct a defect in the tibia, it is usually fractured as well in tibial fractures and the blood supply can be damaged in the face of significant trauma. Bone transport can be utilized in cases where the defect is of moderate length, ${ }^{12}$ but some have 


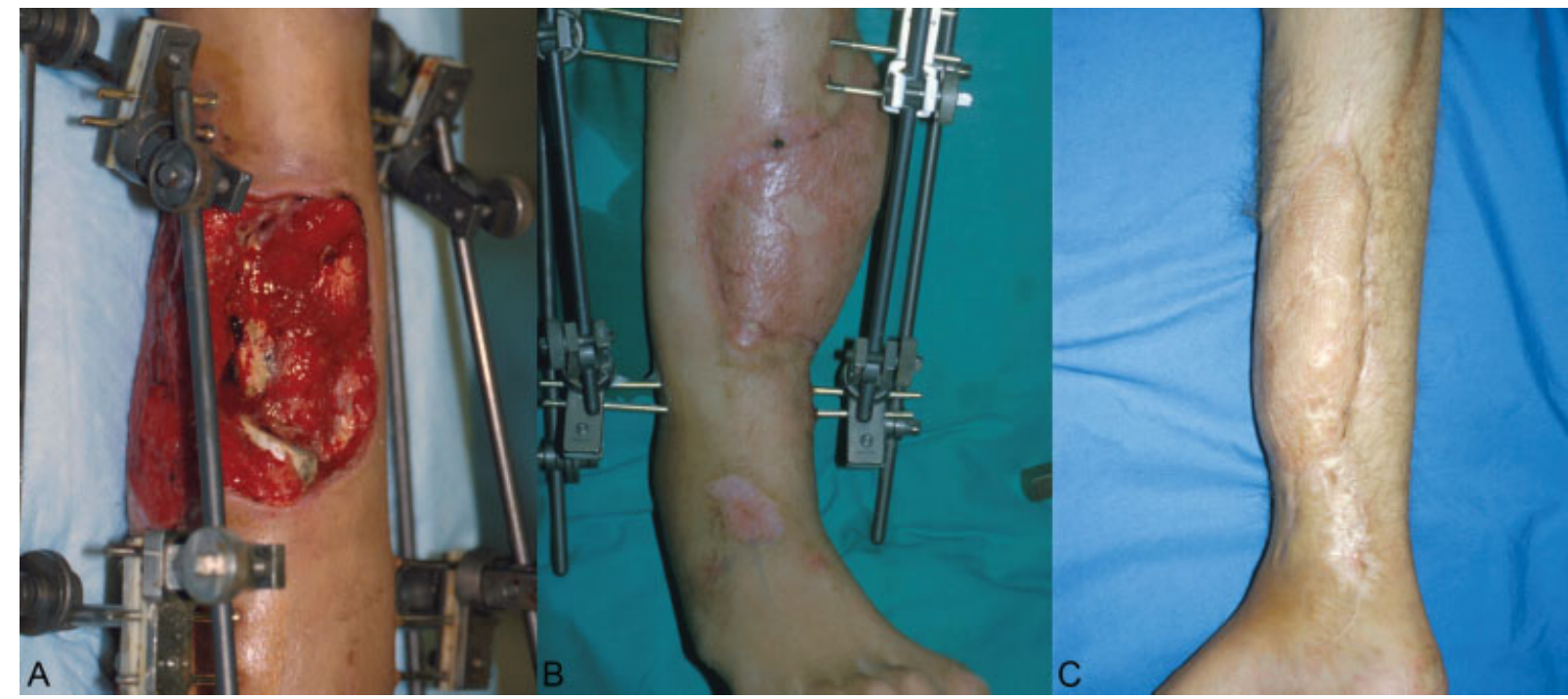

Fig. 1 (A) View of anterior lower leg s/p open fracture. (B) View immediately after microvascular latissimus transfer. (C) View 1 and 1.5 years after muscle flap with split-thickness skin graft. s/p, status post.

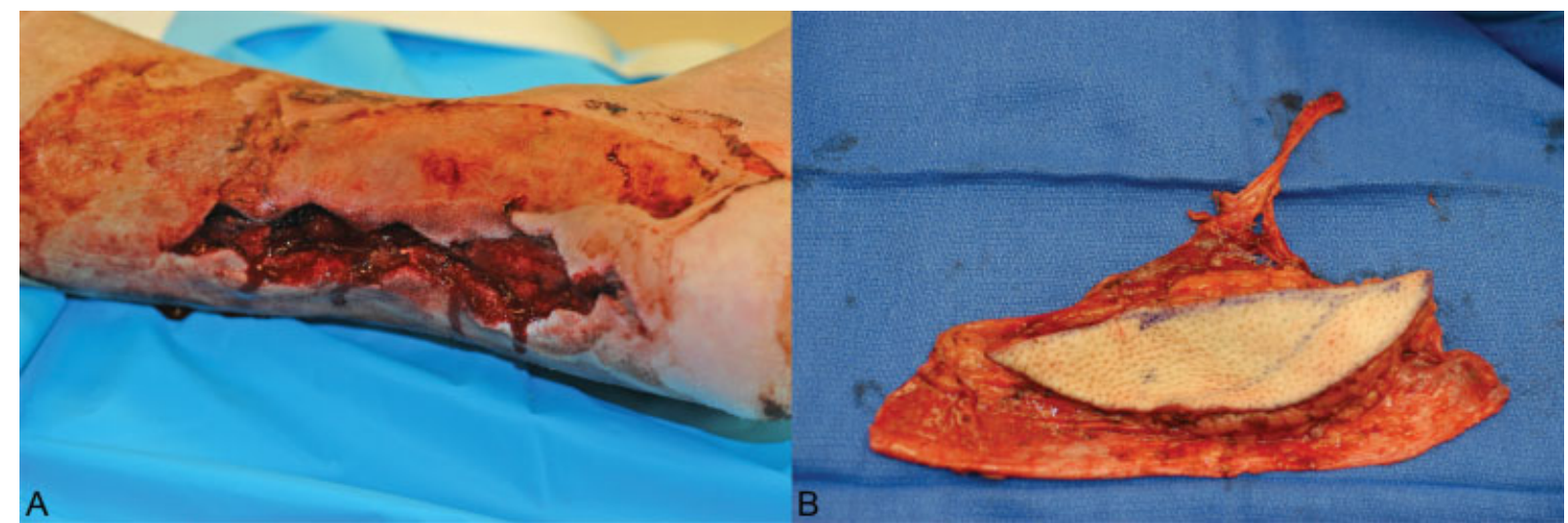

Fig. 2 (A) View of ankle after attempted reconstruction of rerupture of Achilles with allograft and subsequent infection. (B) View of anterolateral thigh flap with a portion of fascia lata to reconstruct the Achilles tendon.

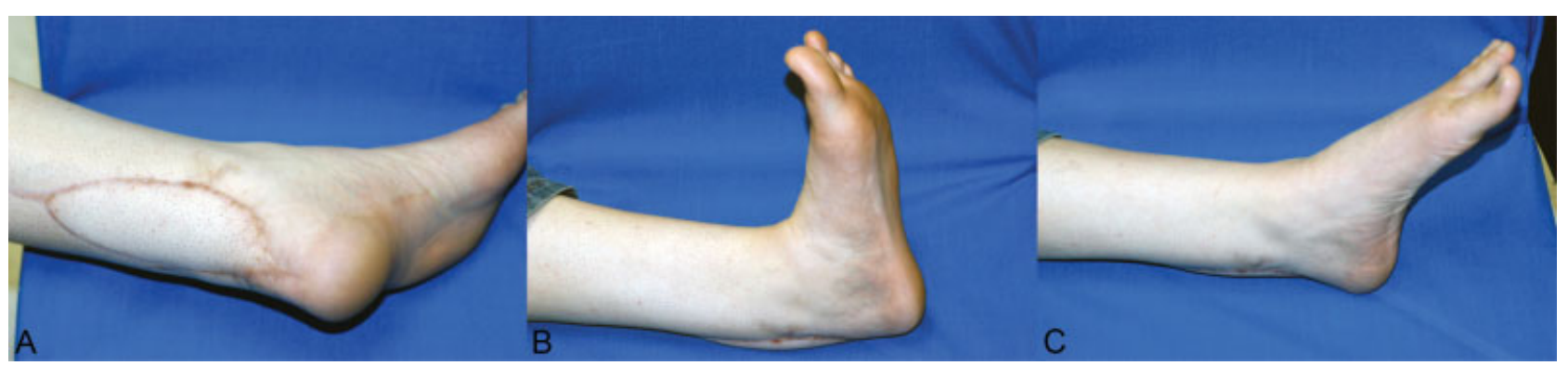

Fig. 3 (A) View of posterior ankle with skin paddle 6 months after reconstruction of Achilles and soft tissue defect. (B) View of ankle extension. (C) View of ankle flexion.

suggested that $12 \mathrm{~cm}$ is the reasonable limit for this approach. $^{13}$

When performing microvascular transfer of the fibula to the tibia, it is generally wise to include a skin paddle. ${ }^{11}$ There is often a soft tissue deficit as discussed earlier, and even if one has previously performed soft tissue reconstruction, the skin with the bone can ensure adequate soft tissue for closure over the bone and also make monitoring the blood flow to the bone possible. We prefer to stabilize the bone with an external fixator and slot the fibula into the tibia if possible, using minimal fixation on the fibula. ${ }^{10,14}$ Multiple drill holes in the fibula should be avoided, as this can compromise the circulation to the bone. Even with vascularized bone, healing can be slow in such complex reconstructions. Healing is not usually adequate for weight-bearing for about 6 months, ${ }^{10}$ and even then the leg needs to be protected in a cast or boot. 


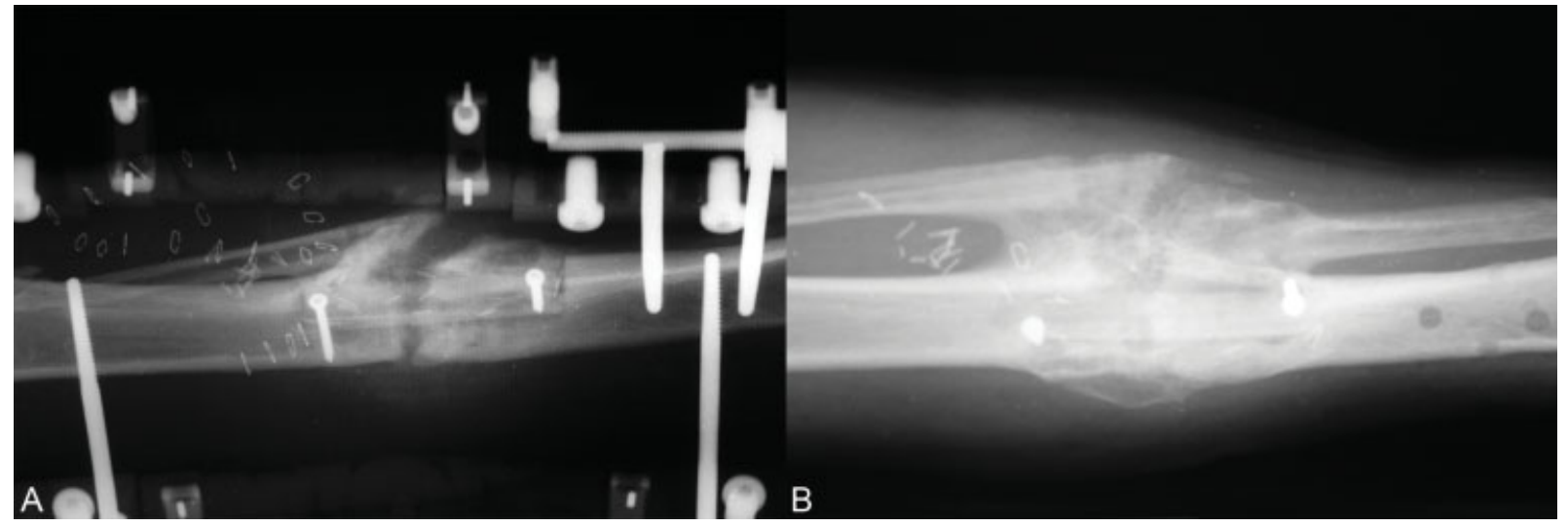

Fig. 4 (A) X-ray of fibula after placement in tibia for chronic nonunion after several operative interventions. (B) X-ray of tibia 6 months after microvascular free fibula transfer.

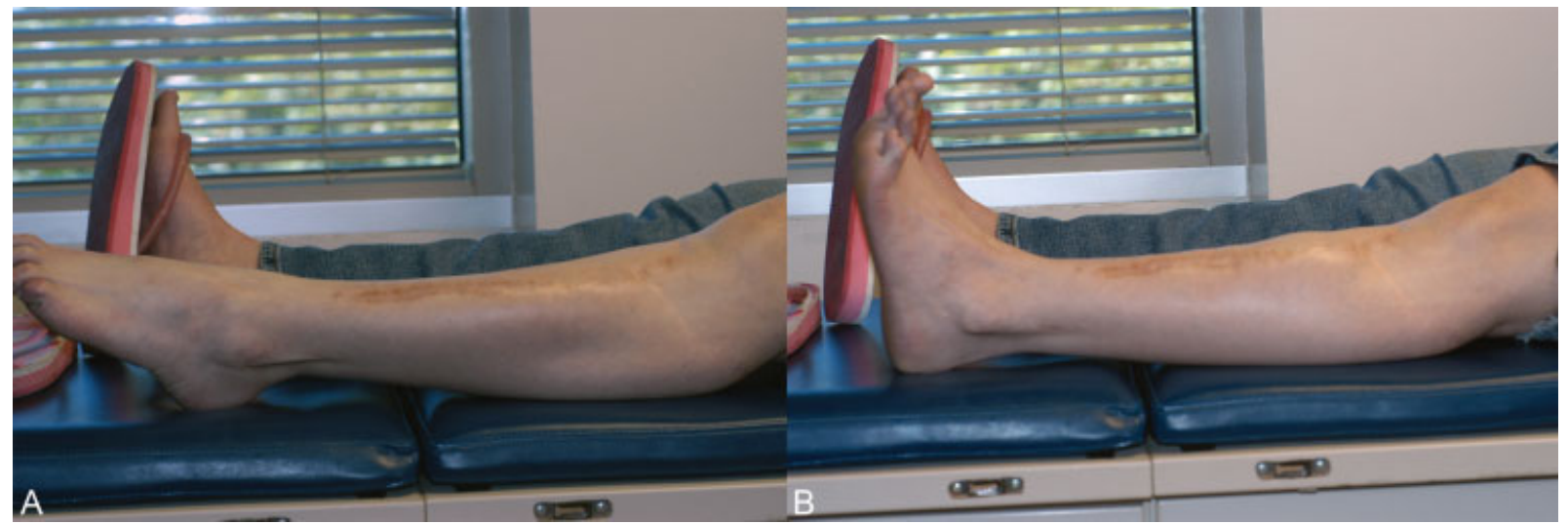

Fig. 5 (A) View of 18-year-old female 5 years after reconstruction of necrotic anterior compartment with innervated free gracilis transfer, flexion. (B) Extension.

Regardless, harvest of the fibula is usually well tolerated in the donor leg. The main morbidities are flexion contracture of the great toe (due to fibrosis of the flexor hallucis longus) and occasional ankle pain. ${ }^{15,16}$

\section{Functional Reconstruction}

Loss of the muscles of the anterior compartment either due to direct trauma or ischemic muscle loss leads to foot drop and the potential need for an ankle foot orthosis for walking. This muscle loss can be improved with tendon transfers, but innervated muscle transfer with a gracilis muscle can provide both coverage and function. While this is a somewhat complex procedure, the gracilis does have adequate length and strength to function as a foot dorsiflexor if placed appropriately in the anterior compartment. The requirements for this to succeed include an adequate tendon distally, an appropriate motor nerve (i.e., the peroneal proximally) for reinnervation, and vessels available for anastomosis (usually the anterior tibial). If these parameters are met, functional reconstruction of foot dorsiflexion can be accomplished (-Fig. 5). When performing innervated microvascular gracilis transfer, the muscle tension must be set properly or the muscle cannot function. The muscle can recover from being placed in too tight, but the actin and myosin fibers cannot recover if they are placed too loosely. The setting of this tension is difficult, but in general, the muscle is marked at $5 \mathrm{~cm}$ intervals with the hip abducted. When it is moved to the leg, it is placed in a stretched position with the markers again placed at $5 \mathrm{~cm}$. Also, the peroneal nerve beyond the fibular neck is mostly motor fibers, particularly in the cephalad portion. This is where the nerve anastomosis should be performed. Even if the nerve anastomosis is done as closely as possible to the muscle, it usually takes a minimum of 6 months before the muscle starts contracting.

\section{Conclusion}

Recent reviews concerning long-term results of microsurgical reconstruction of the lower extremity show good longterm results and reasonable rates of limb salvage. Flap choice does not seem to make much difference in long-term outcome. ${ }^{7,9,17,18}$ Most studies would agree that the deciding factor in outcome is the severity of the injury rather than the choices made in reconstruction.

Conflict of Interest

None declared. 


\section{References}

1 Yazar S, Lin C-H, Wei F-C. One-stage reconstruction of composite bone and soft-tissue defects in traumatic lower extremities. Plast Reconstr Surg 2004;114(06):1457-1466

2 Godina M. Early microsurgical reconstruction of complex trauma of the extremities. Plast Reconstr Surg 1986;78(03):285-292

3 Qiu E, Kurlander DE, Ghaznavi AM. Godina revisited: a systematic review of traumatic lower extremity wound reconstruction timing. J Plast Surg Hand Surg 2018;52(05):259-264

4 Raju A, Ooi A, Ong YS, Tan BK. Traumatic lower limb injury and microsurgical free flap reconstruction with the use of negative pressure wound therapy: is timing crucial? J Reconstr Microsurg 2014;30(06):427-430

5 Isenberg JS, Sherman R. Zone of injury: a valid concept in microvascular reconstruction of the traumatized lower limb? Ann Plast Surg 1996;36(03):270-272

6 Momeni A, Lanni MA, Levin LS, Kovach SJ. Does the use of arteriovenous loops increase complications rates in posttraumatic microsurgical lower extremity reconstruction?-A matched-pair analysis Microsurgery 2018;38(06):605-610

7 Philandrianos C, Moullot P, Gay AM, et al. Soft tissue coverage in distal lower extremity open fractures: comparison of free anterolateral thigh and free latissimus dorsi flaps. J Reconstr Microsurg 2018;34(02):121-129

8 Cho EH, Shammas RL, Carney MJ, et al. Muscle versus fasciocutaneous free flaps in lower extremity traumatic reconstruction: a multicenter outcomes analysis. Plast Reconstr Surg 2018;141(01):191-199

9 Stranix JT, Lee Z-H, Jacoby A, et al. Forty years of lower extremity take-backs: flap type influences salvage outcomes. Plast Reconstr Surg 2018;141(05):1282-1287
10 Pannunzio ME, Chhabra AB, Golish SR, Brown MR, Pederson WC. Free fibula transfer in the treatment of difficult distal tibia fractures. J Reconstr Microsurg 2007;23(01):11-18

11 Henry SL, Frome BA, Pederson WC. Vascularized bone transfer for severe injury around the ankle. Microsurgery 2009;29(05): 353-360

12 Rigal S, Merloz P, Le Nen D, Mathevon H, Masquelet A-C; French Society of Orthopaedic Surgery and Traumatology (SoFCOT). Bone transport techniques in posttraumatic bone defects. Orthop Traumatol Surg Res 2012;98(01):103-108

13 El-Gammal TA, Shiha AE, El-Deen MA, et al. Management of traumatic tibial defects using free vascularized fibula or Ilizarov bone transport: a comparative study. Microsurgery 2008;28(05): 339-346

14 Pederson WC, Person DW. Long bone reconstruction with vascularized bone grafts. Orthop Clin North Am 2007;38(01):23-35, v

15 Rendenbach C, Rashad A, Hansen L, et al. Functional donor site morbidity longer than one year after fibula free flap: a prospective biomechanical analysis. Microsurgery 2018;38(04):395-401

16 Momoh AO, Yu P, Skoracki RJ, Liu S, Feng L, Hanasono MM. A prospective cohort study of fibula free flap donor-site morbidity in 157 consecutive patients. Plast Reconstr Surg 2011;128(03): 714-720

17 Mahajan RK, Srinivasan K, Bhamre A, Singh M, Kumar P, Tambotra A. A retrospective analysis of latissimus dorsi-serratus anterior chimeric flap reconstruction in 47 patients with extensive lower extremity trauma. Indian J Plast Surg 2018;51(01):24-32

18 Bigdeli AK, Gazyakan E, Schmidt VJ, et al. Long-term outcome after successful lower extremity free flap salvage. J Reconstr Microsurg 2018;(October): . Doi: 10.1055/s-0038-1675146 